\title{
Evaluation of the anesthetic efficacy of inferior alveolar nerve blocks in dental patients - A Systematic Review
}

\author{
James D Raj ${ }^{1}$ Sindhu Ramesh ${ }^{2}$ \\ Department of Conservative Dentistry and Endodontics, Saveetha Dental College,Saveetha University, India
}

\begin{abstract}
The inferior alveolar nerve (IAN) block is the most frequently used mandibular injection technique for achieving local anesthesia for dental treatment. However, the IAN block does not always result in successful anesthesia. Various other nerve blocks were introduced over the period of time to improve the success rate of anesthesia.
\end{abstract}

The objective of this systematic review was to compare and evaluate the anesthetic efficacy of Inferior alveolar nerve block with various mandibular nerve blocks in dental patients.

Electronicdatabases were systematically searched for randomized controlled clinical studies and Clinical trials studies. Studies were selected by predefined inclusion criteria. Methodological quality was appraised and strength of evidence was determined.

Seven studies from seven countries were included based on inclusion criteria. Although there is difference in the values comparing the different techniques the data is not significantly different in the anesthetic efficacy of various mandibular nerve block in dental patients.

Based on this review, most of the articles included, point towards a better anesthetic efficacy of the classic inferior alveolar nerve block compared to the other inferior alveolar anesthesia techniques. However due to various variables like type of local anesthesia, experience of the operator and familiarity with the individual techniques it is not possible to conclude that classic inferior alveolar nerve block is relatively superior. Hence further research should be aimed at better matching of groups and variables like operator experience and familiarity to validate the findings.

Keywords: anesthetic efficacy,local anesthesia, local anesthetic technique,mandibular anesthesia,pulpal anesthesia

\section{Introduction}

Successful local anesthesia is the bedrock of pain control in dentistry. Effective pain control is essential to reduce fear and anxiety associated with dental procedures. The inferior alveolar nerve block (IANB) is the conventional method for anesthetizing mandibular teeth. Clinical studies have demonstrated significant failure rates of inferior alveolar nerve block technique, which indicates even if applied appropriately, do not always result in successful anesthesia. This failure rate of IAN blocks represents a common clinical problem for the treatment of mandibular teeth. Supplemental injections (with different techniques and/or types of anesthetic) are frequently required in certain cases to achieve complete anesthesia.

"Gow-Gates[1] introduced a new technique for mandibular anesthesia in 1973." The injection uses extra oral landmarks, and the target site is the neck of the mandibular condyle. A number of studies have shown higher success rates with the Gow-Gates technique (92\%-100\%) than the conventional inferior alveolar nerve technique $(65 \%-86 \%)$. However, "Todorovic et al[2]found a higher success rate with the conventional inferior alveolar nerve block than the Gow-Gates block", whereas "(Ågren and Danielsson[3], Montagnese et al[4], and Hung et al[5]) found the 2 techniques were equivalent."

"Akinosi [6] introduced his technique for mandibular anesthesia in 1977." However, Vazirani also described a similar technique in 1960; hence the name was changed to the Vazirani-Akinosi technique. The injection is a closed mouth technique, with the landmarks for needle insertion being the mucogingival junction of the maxillary second molar. This technique is indicated when there is limited mandibular opening, for example trismus, which precludes the use of the inferior alveolar or Gow-Gates techniques. "(Sisk et al [7] and Todorovic [2]) found the Vazirani-Akinosi technique was equivalent to the conventional inferior alveolar nerve block." However, "(Donkor et al [8], Yücel et al [9], and Gonzales et al [10])" found the conventional IANB was superior to the Vazirani-Akinosi.

\section{Methods}

\section{Database:}

- Electronic search done in Pubmed

- Medline

- Cochrane 


\subsection{Structured Question}

Is there a difference in anesthetic efficacy of Inferior alveolar nerve block when compared to various mandibular blocks in achieving anesthesia in dental patients?

- Population- Dental patients

\section{Pico Analysis}

- Intervention- Inferior alveolar nerve block

- Comparison- Various nerve block techniques of mandible

- Outcome- Anesthetic efficacy

\section{Search Strategy}

A search was performed in electronic database (i.e PUBMED CENTRAL and Medline) using following search terms alone and in combination by means of PUBMED search builder upto June 2012.

\section{Selection Criteria}

Trials were selected if they met the following criteria's: Randomized controlled clinical trials comparing the anesthetic efficacy of Inferior alveolar nerve block with various mandibular nerve blocks in dental patients were included for assessment.

\section{Data Collection and Analysis}

All the studies included were based on the data extraction and analysis of the studies for quality and publication bias. The data collection form was customized. The primary outcome measure was anesthetic success after block administration in dental patients.

\section{FLOWCHART}

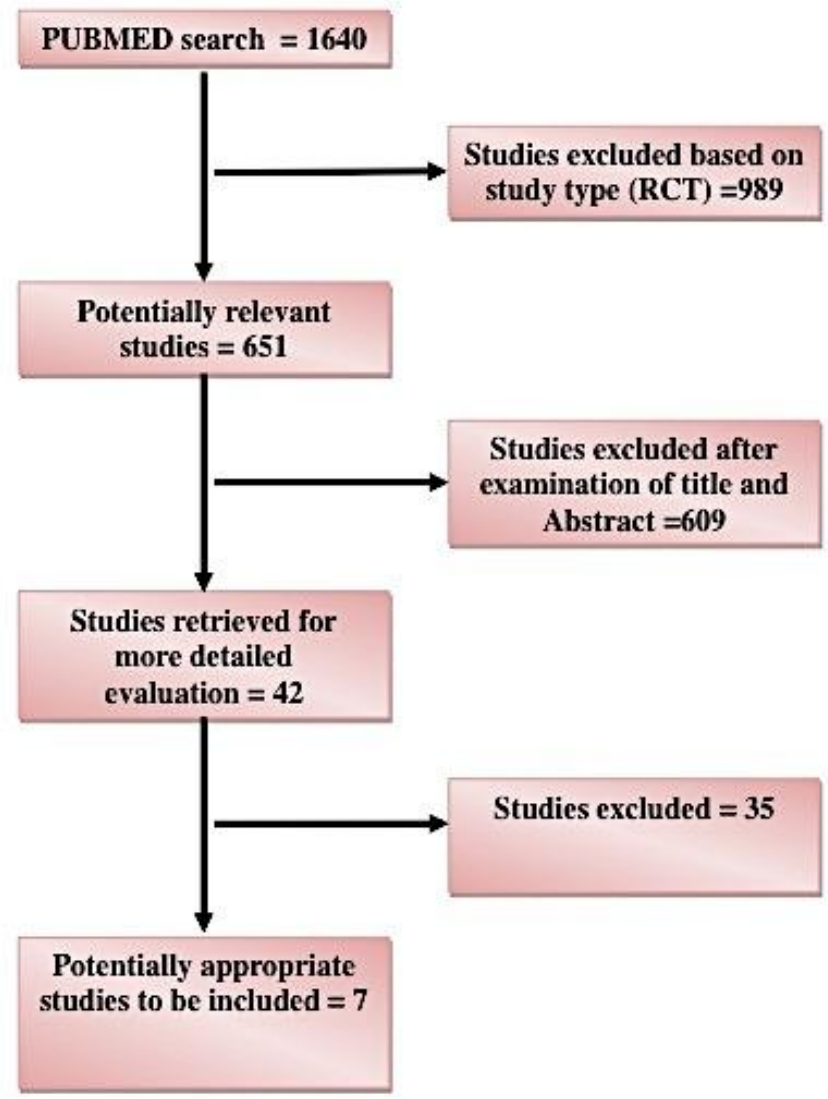




\section{Tables}

Table 1: Variables of interest

\begin{tabular}{|l|l|}
\hline S.No & Variables Of Interest \\
\hline 1 & Anesthetic success \\
\hline
\end{tabular}

Table 2: Characteristics of excluded studies

\begin{tabular}{|c|c|c|c|}
\hline $\begin{array}{l}\text { S } \\
\text { No }\end{array}$ & Author & Year & Reason for Exclusion \\
\hline 1 & $\begin{array}{l}\text { Kämmerer PW, Palarie V, Daubländer M, } \\
\text { Bicer C, Shabazfar N, Brüllmann D, Al-Nawa }\end{array}$ & 2012 & $\begin{array}{l}2 \text { different formulations compared with same } \\
\text { technique }\end{array}$ \\
\hline 2 & Kanaa MD, Whitworth JM, Meechan JG. & 2012 & $\begin{array}{l}\text { Different formulations and supplementary } \\
\text { techniques assessed }\end{array}$ \\
\hline 3 & $\begin{array}{l}\text { Poorni S, Veniashok B, Senthilkumar AD, } \\
\text { Indira R, Ramachandran S. }\end{array}$ & 2011 & Comparison between block and infiltration. \\
\hline 4 & $\begin{array}{l}\text { Kämmerer PW, Palarie V, Daubländer M, } \\
\text { Bicer C, Shabazfar N, Brüllmann D, Al- } \\
\text { NawasB. }\end{array}$ & 2011 & $\begin{array}{l}\text { Anesthetic efficacy with or without } \\
\text { epinephrine analyzed }\end{array}$ \\
\hline 5 & $\begin{array}{l}\text { Martin M, Nusstein J, Drum M, Reader A, Beck } \\
\text { M. }\end{array}$ & 2011 & Different volume of anesthetic agent analyzed \\
\hline 6 & Aggarwal V, Singla M, Rizvi A, Miglani S. & 2011 & $\begin{array}{l}\text { Different formulations and their combination } \\
\text { analyzed }\end{array}$ \\
\hline 7 & $\begin{array}{l}\text { McEntire M, Nusstein J, Drum M, Reader A, } \\
\text { Beck M. }\end{array}$ & 2011 & $\begin{array}{l}\text { Different formulation with difference in } \\
\text { epinephrine concentration }\end{array}$ \\
\hline 8 & $\begin{array}{l}\text { Jaber A, Whitworth JM, Corbett IP, Al-Baqshi } \\
\text { B, Kanaa MD, Meechan JG. }\end{array}$ & 2010 & Different formulations comparing infiltrations \\
\hline 9 & $\begin{array}{l}\text { Nuzum FM, Drum M, Nusstein J, Reader A, } \\
\text { Beck M. }\end{array}$ & 2010 & Combination of infiltration vs single infiltration \\
\hline 10 & $\begin{array}{l}\text { Parirokh M, Satvati SA, Sharifi R, Rekabi AR, } \\
\text { Gorjestani H, Nakhaee N, Abbott PV. }\end{array}$ & 2010 & Combination of buccal infiltration and IANB \\
\hline 11 & $\begin{array}{l}\text { Batista da Silva C, Berto LA, Volpato MC, } \\
\text { Ramacciato JC, Motta RH, Ranali J, Groppo FC. }\end{array}$ & 2010 & $\begin{array}{l}\text { Different agents analyzed for anesthetic } \\
\text { success }\end{array}$ \\
\hline 12 & $\begin{array}{l}\text { Fan S, Chen WL, Pan CB, Huang ZQ, Xian MQ, } \\
\text { Yang ZH, Dias-Ribeiro E, Liang YC,Jiao JY, Ye } \\
\text { YS, } \\
\text { Wen TY }\end{array}$ & 2009 & $\begin{array}{l}\text { Comparison between IANB plus buccal } \\
\text { infiltration vs IANB plus periodontal ligament }\end{array}$ \\
\hline 13 & $\begin{array}{l}\text { Aggarwal V, Jain A, } \\
\text { Kabi D. }\end{array}$ & 2009 & $\begin{array}{l}\text { Buccal and lingual infiltrations compared with } \\
\text { IANB }\end{array}$ \\
\hline 14 & $\begin{array}{l}\text { Kanaa MD, Whitworth JM, Corbett IP, } \\
\text { Meechan JG. }\end{array}$ & 2009 & $\begin{array}{l}\text { Combination of IANB plus Articaine infiltration } \\
\text { analyzed }\end{array}$ \\
\hline 15 & $\begin{array}{l}\text { Tortamano IP, Siviero M, Costa CG, } \\
\text { Buscariolo IA, Armonia PL. }\end{array}$ & 2009 & $\begin{array}{l}\text { Different formulations for anesthetic agent } \\
\text { used analyzed }\end{array}$ \\
\hline 16 & $\begin{array}{l}\text { Goldberg S, Reader A, Drum M, Nusstein J, } \\
\text { Beck M. }\end{array}$ & 2008 & Not in patients but in healthy volunteers \\
\hline 17 & $\begin{array}{l}\text { Haase A, Reader A, Nusstein J, Beck M, Drum } \\
\text { M. }\end{array}$ & 2008 & Different solutions in buccal infiltration \\
\hline 18 & Sherman MG, Flax M, Namerow K, Murray PE. & 2008 & $\begin{array}{l}\text { Maxillary infiltration compared against Gow- } \\
\text { gates block }\end{array}$ \\
\hline 19 & Kohler BR, Castellón L, Laissle G. & 2008 & Different volumes of solution compared \\
\hline 20 & $\begin{array}{l}\text { Whitworth JM, Kanaa MD, Corbett IP, } \\
\text { Meechan JG. }\end{array}$ & 2007 & $\begin{array}{l}\text { Injection speed and anesthetic effectiveness } \\
\text { compared }\end{array}$ \\
\hline 21 & $\begin{array}{l}\text { Robertson D, Nusstein J, Reader A, Beck M, } \\
\text { McCartney M. }\end{array}$ & 2007 & $\begin{array}{l}\text { Buccal infiltration with two different } \\
\text { formulations }\end{array}$ \\
\hline 22 & $\begin{array}{l}\text { Sierra Rebolledo A, Delgado Molina E, } \\
\text { BeriniAytís L, Gay Escoda C. }\end{array}$ & 2007 & Two different formulation analyzed \\
\hline 23 & Bigby J, Reader A, Nusstein J, Beck M. & 2007 & Two different formulation analyzed \\
\hline 24 & $\begin{array}{l}\text { Nusstein J, Steinkruger G, Reader A, Beck M, } \\
\text { Weaver J. }\end{array}$ & 2006 & $\begin{array}{l}\text { 1-stage vs } 2 \text {-stage block, subjects were not } \\
\text { patients }\end{array}$ \\
\hline 25 & $\begin{array}{l}\text { Lai TN, Lin CP, Kok SH, Yang PJ, Kuo YS, Lan } \\
\text { WH, Chang HH. }\end{array}$ & 2006 & Only mandibular block analyzed \\
\hline 26 & Clark K, Reader A, Beck M, Meyers WJ. & 2002 & Infiltration and combination analyzed \\
\hline 27 & $\begin{array}{l}\text { Claffey E, Reader A, Nusstein J, Beck M, } \\
\text { Weaver J. }\end{array}$ & 2004 & Two different formulation analyzed \\
\hline
\end{tabular}




\begin{tabular}{|c|l|l|l|}
\hline 28 & $\begin{array}{l}\text { Kennedy S, Reader A, Nusstein J, Beck M, } \\
\text { Weaver J. }\end{array}$ & 2003 & Conventional vs computer assisted \\
\hline 29 & $\begin{array}{l}\text { Dumbrigue HB, Lim MV, Rudman RA, Serraon } \\
\text { A. }\end{array}$ & 1997 & $\begin{array}{l}\text { Nerve block compared with intraligamentary } \\
\text { injection }\end{array}$ \\
\hline 30 & $\begin{array}{l}\text { Dunbar D, Reader A, Nist R, Beck M, Meyers } \\
\text { WJ. }\end{array}$ & 1996 & $\begin{array}{l}\text { Intra osseous injection vs inferior alveolar } \\
\text { nerve block }\end{array}$ \\
\hline 31 & $\begin{array}{l}\text { Childers M, Reader A, Nist R, Beck M, Meyers } \\
\text { WJ. }\end{array}$ & 1996 & $\begin{array}{l}\text { Periodontal ligament injection against inferior } \\
\text { alveolar nerve block }\end{array}$ \\
\hline 32 & $\begin{array}{l}\text { Syverud SA, Jenkins JM, Schwab RA, Lynch } \\
\text { MT, Knoop K, Trott A. }\end{array}$ & 1994 & Healthy volunteers participated in study. \\
\hline 33 & $\begin{array}{l}\text { Zanette G, Manani G, Facco E, Mariuzzi ML, } \\
\text { Tregnaghi A, Robb ND. }\end{array}$ & 2011 & Comparison not relevant \\
\hline 34 & \begin{tabular}{l} 
Takasugi Y, Furuya H, Moriya K, Okamoto Y \\
\hline 35
\end{tabular} & 2000 & Comparison not relevant \\
\hline
\end{tabular}

Table 3: General information of selected articles

\begin{tabular}{|c|c|c|c|c|c|c|c|c|c|}
\hline $\begin{array}{l}\mathrm{S} \\
\mathrm{n} \\
\mathrm{o}\end{array}$ & Author & Year & Country & $\begin{array}{l}\text { Study } \\
\text { design }\end{array}$ & $\begin{array}{l}\text { Sample } \\
\text { size }\end{array}$ & Age & Set-up & Techniques used & $\begin{array}{l}\text { Method of } \\
\text { evaluation }\end{array}$ \\
\hline 1 & $\begin{array}{l}\text { Aggarwal } \\
\text { V et al }\end{array}$ & 2010 & India & $\begin{array}{l}\text { RCT } \\
\text { double } \\
\text { blinded } \\
\text { trial }\end{array}$ & 97 & $>18 \mathrm{yrs}$ & University & $\begin{array}{l}\text { Gow-gates, } \\
\text { Vazirani-Akinosi, } \\
\text { Buccal plus Lingual } \\
\text { Infiltrations and } \\
\text { Inferior alveolar } \\
\text { nerve block }\end{array}$ & $\begin{array}{l}\text { Lip numbness } \\
\text { Initial access } \\
\text { opening }\end{array}$ \\
\hline 2 & $\begin{array}{l}\text { Hung PC } \\
\text { et al }\end{array}$ & 2006 & Taiwan & $\begin{array}{l}\mathrm{RCT} \\
\text { double } \\
\text { blinded } \\
\text { trial } \\
\end{array}$ & 162 & $>18 \mathrm{yrs}$ & University & $\begin{array}{l}\text { Gow-gates and } \\
\text { Inferior alveolar } \\
\text { nerve block }\end{array}$ & $\begin{array}{l}\text { Electric pulp } \\
\text { tester and a } \\
\text { sharp explorer }\end{array}$ \\
\hline 3 & $\begin{array}{l}\text { Martínez } \\
\text { González } \\
\text { JM et al }\end{array}$ & 2003 & Spain & $\begin{array}{l}\text { RCT } \\
\text { double } \\
\text { blinded } \\
\text { trial }\end{array}$ & 56 & $>18 \mathrm{yrs}$ & University & $\begin{array}{l}\text { Direct Mandibular } \\
\text { Nerve Block and } \\
\text { Akinosi technique }\end{array}$ & $\begin{array}{l}\text { Lower lip } \\
\text { numbness }\end{array}$ \\
\hline 4 & Yucel et al & 1995 & Turkey & $\begin{array}{l}\text { RCT } \\
\text { double } \\
\text { blinded } \\
\text { trial }\end{array}$ & 69 & $>18 \mathrm{yrs}$ & University & $\begin{array}{l}\text { Direct technique, } \\
\text { and Akinosi } \\
\text { technique }\end{array}$ & $\begin{array}{l}\text { Numbness of } \\
\text { the lower lip } \\
\text { Adequacy of } \\
\text { tissue } \\
\text { anesthesia - } \\
\text { Probing }\end{array}$ \\
\hline 5 & $\begin{array}{l}\text { Waikakul } \\
\text { A et al }\end{array}$ & 1991 & Thailand & $\begin{array}{l}\text { RCT } \\
\text { double } \\
\text { blinded } \\
\text { trial }\end{array}$ & 136 & $>18 y r s$ & University & $\begin{array}{l}\text { Direct technique and } \\
\text { Extra-Intraoral } \\
\text { Landmark (EIL) } \\
\text { technique. }\end{array}$ & $\begin{array}{l}\text { Thickening } \\
\text { sensations on } \\
\text { lower lip, } \\
\text { tongue and } \\
\text { cheek. } \\
\text { Explorer } \\
\text { testing }\end{array}$ \\
\hline 6 & $\begin{array}{l}\text { Donkor P } \\
\text { et al }\end{array}$ & 1990 & Australia & $\begin{array}{l}\text { RCT } \\
\text { double } \\
\text { blinded } \\
\text { trial }\end{array}$ & 200 & $>18 \mathrm{yrs}$ & University & $\begin{array}{l}\text { Closed-mouth and } \\
\text { Conventio-nal Block } \\
\text { Injection Technique }\end{array}$ & $\begin{array}{l}\text { Lip numbness } \\
\text { Probing the } \\
\text { soft tissues }\end{array}$ \\
\hline 7 & $\begin{array}{l}\text { Todorovic } \\
\text { et al }\end{array}$ & 1986 & $\begin{array}{l}\text { Former } \\
\text { Yugoslav } \\
\text { ia }\end{array}$ & $\begin{array}{l}\text { RCT } \\
\text { double } \\
\text { blinded } \\
\text { trial }\end{array}$ & 90 & $>19 y r s$ & University & $\begin{array}{l}\text { Gow-gates, } \\
\text { Vazirani-Akinosi, } \\
\text { and Direct Inferior } \\
\text { Alveolar Nerve } \\
\text { block }\end{array}$ & $\begin{array}{l}\text { Numbness of } \\
\text { lower lip. } \\
\text { Pinprick on } \\
\text { tissue } \\
\text { supplied by } \\
\text { sensory } \\
\text { branches of } \\
\text { mandibular } \\
\text { nerve }\end{array}$ \\
\hline
\end{tabular}


Table 4: Results

\begin{tabular}{|c|c|c|c|c|c|}
\hline $\begin{array}{l}\text { S. } \\
\text { No. }\end{array}$ & Author and Year & Materials used & Method of evaluation & Mean values & Outcome \\
\hline 1. & $\begin{array}{l}\text { Aggarwal V et al } \\
2010\end{array}$ & $\begin{array}{l}\text { Gow-gates, } \\
\text { Vazirani-Akinosi, } \\
\text { Buccal plus } \\
\text { Lingual } \\
\text { Infiltrations and } \\
\text { Inferior alveolar } \\
\text { nerve block }\end{array}$ & $\begin{array}{l}\text { Subjective: } \\
\text { Lip numbness } \\
\text { Objective: } \\
\text { Initial access opening }\end{array}$ & $\begin{array}{l}\text { Gow-gates- 52\% } \\
\text { Vazirani-Akinosi-41\% } \\
\text { Buccal plus Lingual } \\
\text { Infiltrations-27\% } \\
\text { Inferior alveolar nerve } \\
\text { block-36\% }\end{array}$ & $\begin{array}{l}\text { Gow-Gates success rate } \\
\text { of } 52 \% \text {, which was } \\
\text { statistically higher than } \\
\text { control IANB }(36 \%) \text { (P } \\
<.05) \text {. }\end{array}$ \\
\hline 2. & Hung PC et al 2006 & $\begin{array}{l}\text { Gow-gates and } \\
\text { Inferior alveolar } \\
\text { nerve block }\end{array}$ & $\begin{array}{l}\text { Objective: } \\
\text { Electric pulp tester } \\
\text { and a sharp explorer }\end{array}$ & $\begin{array}{l}\text { IANB group } \\
\text { Central Incisor-6\% } \\
\text { Canine-37\% } \\
\text { First PM- 54\% } \\
\text { First Molar- 88\% } \\
\text { GGMB group } \\
\text { Central Incisor- } 8.1 \% \\
\text { Canine-37.1\% } \\
\text { First PM- 54.8\% } \\
\text { First Molar- } 83.9 \%\end{array}$ & $\begin{array}{l}\text { No significant } \\
\text { difference between the } \\
\text { two groups }\end{array}$ \\
\hline 3 & $\begin{array}{l}\text { Martínez González } \\
\text { JM et al } 2003\end{array}$ & $\begin{array}{l}\text { Direct Mandibular } \\
\text { Nerve Block and } \\
\text { Akinosi technique }\end{array}$ & $\begin{array}{l}\text { Subjective: } \\
\text { Lower lip numbness }\end{array}$ & $\begin{array}{l}\text { Success rates } \\
\text { Direct mandibular nerve } \\
\text { block }-100 \% \\
\text { Akinosi technique- } 92 \%\end{array}$ & $\begin{array}{l}\text { Akinosi technique is not } \\
\text { as effective as } \\
\text { conventional direct } \\
\text { mandibular block. }\end{array}$ \\
\hline 4 & Yucel et al 1995 & $\begin{array}{l}\text { Direct technique, } \\
\text { and Akinosi } \\
\text { technique }\end{array}$ & $\begin{array}{l}\text { Subjective: } \\
\text { Numbness of the } \\
\text { lower lip } \\
\text { Objective: } \\
\text { Adequacy of tissue } \\
\text { anesthesia -Probing }\end{array}$ & $\begin{array}{l}\text { Direct technique }-98 \% \\
\text { Akinosi technique }-76 \%\end{array}$ & $\begin{array}{l}\text { The direct technique } \\
\text { was more successful in } \\
\text { achieving inferior } \\
\text { alveolar nerve } \\
\text { anesthesia (SND:3.815; } \\
\text { p<0.001). }\end{array}$ \\
\hline 5 & $\begin{array}{l}\text { Waikakul A et al } \\
1991\end{array}$ & $\begin{array}{l}\text { Direct technique } \\
\text { and Extra-Intraoral } \\
\text { Landmark (EIL) } \\
\text { technique. }\end{array}$ & $\begin{array}{l}\text { Subjective: } \\
\text { Thickening } \\
\text { sensations on lower } \\
\text { lip, tongue and } \\
\text { cheek. } \\
\text { Objective: } \\
\text { Explorer testing on } \\
\text { the lower lip and } \\
\text { labial and lingual } \\
\text { gingiva of canine. }\end{array}$ & $\begin{array}{l}\text { Subjective: } \\
\text { EIL technique-66.2\% } \\
\text { Direct technique- } 47.1 \% \\
\text { Objective: } \\
\text { EIL technique- Labial- } \\
85.3 \% \\
\text { Lingual-89.7\% } \\
\text { Direct technique- } \\
\text { Labial-58.8\% } \\
\text { Lingual-76.5\% }\end{array}$ & $\begin{array}{l}\text { Failure rate of Direct } \\
\text { technique significantly } \\
\text { greater than in the EIL } \\
\text { group }(\mathrm{x} 2=9.68, \mathrm{P} \\
<.05) . \\
\text { Numbness of labial and } \\
\text { lingual gingiva } \\
\text { significantly greater in } \\
\text { the EIL group than the } \\
\text { control group }(\mathrm{x} 2= \\
10.55, \mathrm{P}<.05) .\end{array}$ \\
\hline 6 & $\begin{array}{l}\text { Donkor P et al } \\
1990\end{array}$ & $\begin{array}{l}\text { Closed-mouth and } \\
\text { Conventio-nal } \\
\text { Block Injection } \\
\text { Technique }\end{array}$ & $\begin{array}{l}\text { Subjective: } \\
\text { Lip numbness } \\
\text { Objective: } \\
\text { Probing the soft } \\
\text { tissues }\end{array}$ & $\begin{array}{l}\text { Success rates } \\
\text { Conventional group } 97 \% \\
\text { Closed-Mouth group } 79 \%\end{array}$ & $\begin{array}{l}\text { Conventional technique } \\
\text { more effective than } \\
\text { Closed Mouth } \\
\text { Technique in achieving } \\
\text { anesthesia } \\
(\mathrm{SND}=3.917, \mathrm{p}<0.001) \text {. }\end{array}$ \\
\hline 7 & $\begin{array}{l}\text { Todorovic et al } \\
1986\end{array}$ & $\begin{array}{l}\text { Gow-gates, } \\
\text { Vazirani-Akinosi, } \\
\text { and Direct Inferior } \\
\text { Alveolar Nerve } \\
\text { block }\end{array}$ & $\begin{array}{l}\text { Subjective: } \\
\text { Numbness of lower } \\
\text { lip. } \\
\text { Objective: } \\
\text { Pinprick on tissue } \\
\text { supplied by sensory } \\
\text { branches of } \\
\text { mandibular nerve }\end{array}$ & $\begin{array}{l}\text { Direct method - } 96.6 \% \\
\text { Gow-Gates- } 90 \% \\
\text { Akinosi-76.6\% }\end{array}$ & $\begin{array}{l}\text { Statistical difference } \\
\text { between Direct method } \\
\text { and Akinosi }\end{array}$ \\
\hline
\end{tabular}

Table 5: Summation tables for individual parameter

\begin{tabular}{|l|l|l|l|l|l|l|}
\hline \multirow{2}{*}{$\begin{array}{l}\text { S. } \\
\text { No }\end{array}$} & \multirow{2}{*}{ Author } & & \multicolumn{5}{|c|}{ Outcome Anesthetic Success (\%) } \\
\cline { 4 - 7 } & & & IANB & GOW-GATES & $\begin{array}{l}\text { VAZIRANI } \\
\text { AKINOSI }\end{array}$ & OTHERS \\
\hline 1. & Aggarwal et al & 2010 & $36 \%$ & $52 \%$ & $41 \%$ & $27 \%$ \\
\hline 2. & Hung PC et al & 2006 & $88 \%$ & $83.9 \%$ & - & - \\
\hline 3. & González et al & 2003 & $100 \%$ & - & $92 \%$ & - \\
\hline 4. & Yucel et al & 1995 & $98 \%$ & - & $76 \%$ & - \\
\hline 5. & WaikakulA et al & 1991 & $67.65 \%$ & - & - & $87.5 \%$ \\
\hline 6. & Donkor P et al & 1990 & $97 \%$ & - & $79 \%$ & - \\
\hline 7. & Todorovic et al & 1986 & $96.6 \%$ & $90.6 \%$ & $76.6 \%$ & - \\
\hline
\end{tabular}


Table 6: Evidence level of selected articles

\begin{tabular}{|l|l|l|l|l|}
\hline S.No & \multicolumn{1}{|c|}{ Author } & Year & \multicolumn{1}{|c|}{ Study Design } & $\begin{array}{l}\text { Level of } \\
\text { Evidence }\end{array}$ \\
\hline 1 & Aggarwal V & 2010 & $\begin{array}{l}\text { Randomized double blinded clinical } \\
\text { trial }\end{array}$ & Level 2 \\
\hline 2 & Hung PC & 2006 & Randomized controlled clinical trial & Level 2 \\
\hline 3 & Martínez González JM & 2003 & Randomized clinical trial & Level 2 \\
\hline 4 & Yucel M & 1995 & $\begin{array}{l}\text { Randomized double blinded controlled } \\
\text { clinical trial }\end{array}$ & Level 2 \\
\hline 5 & Waikakul A & 1991 & $\begin{array}{l}\text { Randomized double blinded clinical } \\
\text { trial }\end{array}$ & Level 2 \\
\hline 6 & Donkor P & 1990 & $\begin{array}{l}\text { Randomized double blinded clinical } \\
\text { trial }\end{array}$ & Level 2 \\
\hline 7 & Todorovic & 1986 & Randomized clinical trial & Level 2 \\
\hline
\end{tabular}

Table 7: Risk of bias - major criteria

\begin{tabular}{|l|l|l|l|l|c|}
\hline \multicolumn{1}{|c|}{ Study } & $\begin{array}{l}\text { Random } \\
\text {-ization }\end{array}$ & $\begin{array}{l}\text { Allocation } \\
\text { Concealed }\end{array}$ & $\begin{array}{l}\text { Assessor } \\
\text { Blinding }\end{array}$ & $\begin{array}{l}\text { Dropouts } \\
\text { Described }\end{array}$ & $\begin{array}{l}\text { Risk of } \\
\text { Bias }\end{array}$ \\
\hline Aggarwal V et al & No & No & No & None & High \\
\hline Hung PC et al & No & No & No & None & High \\
\hline González JM et al & Yes & No & No & None & Mod \\
\hline Yucel et al & Yes & No & Yes & None & Low \\
\hline Waikakul A et al & No & No & Yes & None & Mod \\
\hline Donkor P et al & Yes & No & Yes & None & Low \\
\hline Todorovic et al & No & No & No & None & High \\
\hline
\end{tabular}

Table 8: Risk of bias - minor criteria

\begin{tabular}{|l|l|l|l|l|}
\hline Study & Sample Justified & Baseline comparison & I/ E Criteria & Method Error \\
\hline Aggarwal V et al & Yes & Yes & Yes & No \\
\hline Hung PC et al & No & Yes & Yes & No \\
\hline González et al & No & Yes & Yes & No \\
\hline Yucel et al & No & Yes & Yes & No \\
\hline Waikakul A et al & No & Yes & Yes & No \\
\hline Donkor P et al & No & Yes & Yes & No \\
\hline Todorovic et al & No & No & No \\
\hline
\end{tabular}

\section{Description of Studies}

\section{Results}

The search identified 1640 publications out of which 989 were excluded after applying limits for randomized controlled clinical trial. Further 609 were excluded from 651 after reviewing the title or abstract based on relevance to our topic. Full articles were obtained for 42 studies 35 of these publications were excluded after reading the full text article. Therefore, a total of 7 publications fulfilled all criteria for inclusion.

Although there is difference in the values comparing the different techniques the data is not significantly different in the anesthetic efficacy of various mandibular nerve block in dental patients.

Aggarwal V et al [11] included 97 adult subjects, who were actively experiencing pain, participated in prospective, randomized, double-blinded study. Twenty-five patients received Gow-Gates mandibular conduction block anesthesia, 24 patients received "high" Vazirani-Akinosi inferior alveolar nerve block, 26 received only buccal-plus-lingual infiltrations, and 22 patients (control) received conventional IANB anesthesia.

Endodontic access preparation was initiated after 15 minutes of anesthesia. Pain during treatment was recorded using a Heft-Parker visual analog scale. Success was recorded for "none" or "mild" pain. Gow-Gates gave a success rate of 52\%, which was statistically higher than control IANB (36\%) (P < .05). Vazirani-Akinosi and infiltrations gave $41 \%$ and $27 \%$ success rates, respectively, with no statistically significant differences from control IANB.

Hung et al [5] 162 patients (93 males and 69 females) who were randomly allocated to receive Gow Gates or IANB for extraction of third molars. Both methods used $2.7 \mathrm{~mL}$ of $2 \%$ xylocaine for each patient. Pulpal and gingival tissue anesthesia of mandibular central incisors, canines, first premolars and first molars were evaluated at $0,5,10,15$ and 60 minutes after injection of local anesthetic solution using both an electric pulp tester and a sharp explorer. The success rates of pulpal anesthesia in the IANB group (central incisor, $6 \%$; canine, 37\%; first premolar, 54\%; first molar, 88\%) were not significantly different from the GGMB group (central incisor, 8.1\%; canine, 37.1\%; first premolar, 54.8\%; first molar, 83.9\%). The efficacy of pulpal and gingival tissue anesthesia is not significantly different between the GGMB and IANB methods.

Martínez González JM et al [10] included 56 patients programmed for lower third molar extraction. 22 males and 34 females were randomly assigned to two groups of 28 subjects each: control (subjected to direct 
mandibular nerve block) and study group (anesthesia with the Akinosi technique). The same anesthetic solution was used in all cases, i.e., a cartridge containing $1.8 \mathrm{ml}$ of $4 \%$ articaine with epinephrine 1:100,000. The statistical analysis of the results was based on the comparison of means for quantitative variables using the Student t-test, with the chi-square test for the comparison of qualitative variables. Comparison between groups was done using ANOVA. Anesthetic effect was achieved in all of the patients subjected to conventional mandibular nerve block, versus in $92 \%$ of those subjected to the Akinosi technique.

Yucel et al [9] included 250 patients of both sexes requiring the extraction of lower first or second molar teeth. Patients were divided into two groups: a control group underwent the direct technique, and the Akinosi technique was used on the experimental group. In all cases injections of $2 \mathrm{~mL}$ of lignocaine hydrochloride with 1:200 000 epinephrine hydrochloride were given with 27 -gauge, $50 \mathrm{~mm}$ long disposable needles. Results of the study were statistically analyzed using the Student's t test and the standard normal deviate test (SND). The direct technique was more successful in achieving inferior alveolar nerve anesthesia (SND: $3.815 ; \mathrm{p}<0.001$ ).

Waikakul A et al [12] 136 Patients who came to the Oral Surgery Clinic, Faculty of Dentistry, Mahidol University for mandibular tooth extraction were selected for this study. They were divided equally into two groups: a control group that underwent the direct technique as described by Bennett and the experimental group, with which the EIL technique was used. The local anesthetic solution used was $1.8 \mathrm{~mL}$ of $2 \%$ lidocaine HCI with 1:100,000 epinephrine injected with a 27 -gauge, $30-\mathrm{mm}$-long disposable needle. The randomized matching of the operators and techniques was done just prior to the injection by the first investigator. Second investigator, not aware of which technique had been used, recorded the patient's feelings concerning the onset of tingling or thickening sensations on the lower lip, tongue and cheek.

Donkor et al [8] included 200 patients, who were randomly allotted to the closed mouth and conventional mandibular technique. Lip numbness was reported as early as $5 \mathrm{~min}$ or between $5-10 \mathrm{~min}$.if no change in lip sensation after 10min supplementary block injection given using same technique. Probing the soft tissues supplied by branches of inferior alveolar, lingual and long buccal nerves tested anesthetic efficacy.

Anesthesia was considered successful if no pain was reported on probing. The conventional technique was significantly more successful in achieving inferior alveolar nerve anesthesia $(\mathrm{p}<0.001)$.

Todorovic et al [2] included 90 patients of both sexes, aged from 17 to 62 years, undergoing simple tooth extraction into his prospective study. They were randomly allocated into 3 groups with regard to the applied technique of mandibular anesthesia. In all cases, injections of $2 \mathrm{ml}$ of $2 \%$ lidocaine with adrenaline $(1: 80,000)$, using disposable syringes, were given by the authors. The results were statistically analyzed using the chi square test.

\section{Defending the Results}

From this review classic inferior alveolar nerve block/Direct Technique is shown to provide better anesthetic efficacy compared to other techniques. This could be attributed to more practitioners being familiar with this technique as it is the commonest technique taught in dental schools.

Compared to other methods inferior alveolar nerve block easier to learn due to the numerous traceable landmarks. Also even though positive aspiration is higher in IANB clinically evident hematoma formation is lower compared to other methods, thus it is safer for clinical applications. Although not all articles reported statistically significant differences between the various techniques, they do however report a higher level of anesthetic efficacy with classic inferior alveolar nerve block.

\section{Inference}

\section{Implications for Practice}

Anesthetic success depends on familiarity of the practitioner with one technique. Based on this review the evidence points more towards the experience of the operator rather than technique.

\section{Implications for Research}

In future research should be aimed at better matching of groups and variables like operator experience and familiarity to validate the findings.

\section{Conclusion}

Based on this review,most of the articles included point towards a better anesthetic efficacy of the classic inferior alveolar nerve block compared to the other inferior alveolar anesthesia techniques. However due to various variables like type of local anesthesia, experience of the operator and familiarity with the individual techniques it is not possible to conclude that classic inferior alveolar nerve block is infact superior.

Hence further research should be aimed at better matching of groups and variables like operator experience and familiarity to validate the findings. 


\section{References}

[1]. Gow-Gates, G. A., and J. E. Watson. "The Gow-Gates mandibular block: further understanding." Anesthesia Progress 24.6 (1977): 183.

[2]. Todorović L, Stajcić Z, Petrović V. Mandibular versus inferior dental anaesthesia: clinical assessment of 3 different techniques.Int $\mathbf{J}$ Oral Maxillofac Surg. 1986 Dec; 15(6): 733-8.

[3]. Agren, E., and K. Danielsson. "Conduction block analgesia in the mandible. A comparative investigation of the techniques of Fischer and Gow-Gates." Swedish dental journal 5.3 (1981): 81.

[4]. Montagnese, Thomas A., Al Reader, and Rudy Melfi. "A comparative study of the Gow-Gates technique and a standard technique for mandibular anesthesia."Journal of endodontics 10.4 (1984): 158-163.

[5]. Hung PC, Chang HH, Yang PJ, Kuo YS, Lan WH, Lin CP. Comparison of the Gow-Gates mandibular block and inferior alveolar nerve block using a standardized protocol.JFormos Med Assoc. 2006 Feb; 105(2): 139-46.

[6]. Akinosi, J. O. "A new approach to the mandibular nerve block. "British Journal of Oral Surgery 15.1 (1977): 83-87.

[7]. Sisk, Allen L. "Evaluation of the Akinosi mandibular block technique in oral surgery."Journal of Oral and Maxillofacial Surgery 44.2 (1986): 113-115.

[8]. Donkor P, Wong J, Punnia-Moorthy A. An evaluation of the closed mouth mandibular block technique. Int J Oral Maxillofac Surg. 1990 Aug; 19(4): 216-9.

[9]. Yücel E, Hutchison IL. A comparative evaluation of the conventional and closed-mouth technique for inferior alveolar nerve block.Aust Dent J. 1995Feb; 40(1): 15-6.

[10]. Martínez González JM, Benito Peña B, FernándezCáliz F, San HipólitoMarín L, PeñarrochaDiago M. A comparative study of direct mandibular nerve block and the Akinosi technique. Med Oral. 2003 Mar-Apr;8(2): 143-9. English, Spanish.

[11]. Aggarwal V, Singla M, Kabi D. Comparative evaluation of anesthetic efficacy of Gow-Gates mandibular conduction anesthesia, Vazirani-Akinosi technique, buccal-plus-lingual infiltrations, and conventional inferior alveolar nerve anesthesia in patients with irreversible pulpitis.OralSurg Oral Med Oral PatholOralRadiolEndod. 2010 Feb; 109(2): 303-8.

[12]. Waikakul A, Punwutikorn J. A comparative study of the extra-intraoral landmark technique and the direct technique for inferior alveolar nerve block. J Oral Maxillofac Surg. 1991 Aug; 49(8): 804-8; discussion 808-9. 